\title{
An explicit approximate solution to the Duffing- harmonic oscillator by a cubication method
}

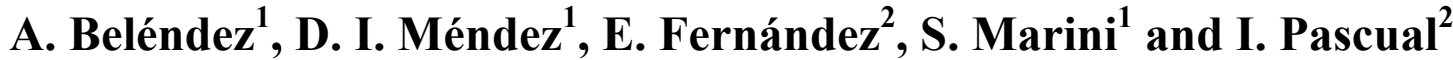

${ }^{1}$ Departamento de Física, Ingeniería de Sistemas y Teoría de la Señal.

Universidad de Alicante. Apartado 99. E-03080 Alicante. SPAIN

${ }^{2}$ Departamento de Óptica, Farmacología y Anatomía.

Universidad de Alicante. Apartado 99. E-03080 Alicante. SPAIN

E-mail: a.belendez@ua.es

Corresponding author: A. Beléndez

Phone: +34-96-5903651

Fax: $+34-96-5909750$

E-mail: a.belendez@ua.es 


\begin{abstract}
The nonlinear oscillations of a Duffing-harmonic oscillator are investigated by an approximated method based on the 'cubication' of the initial nonlinear differential equation. In this cubication method the restoring force is expanded in Chebyshev polynomials and the original nonlinear differential equation is approximated by a Duffing equation in which the coefficients for the linear and cubic terms depend on the initial amplitude, $A$. The replacement of the original nonlinear equation by an approximate Duffing equation allows us to obtain explicit approximate formulas for the frequency and the solution as a function of the complete elliptic integral of the first kind and the Jacobi elliptic function, respectively. These explicit formulas are valid for all values of the initial amplitude and we conclude this cubication method works very well for the whole range of initial amplitudes. Excellent agreement of the approximate frequencies and periodic solutions with the exact ones is demonstrated and discussed and the relative error for the approximate frequency is as low as $0.071 \%$. Unlike other approximate methods applied to this oscillator, which are not capable to reproduce exactly the behaviour of the approximate frequency when $A$ tends to zero, the cubication method used in this paper predicts exactly the behaviour of the approximate frequency not only when $A$ tends to infinity, but also when $A$ tends to zero. Finally, a closedform expression for the approximate frequency is obtained in terms of elementary functions. To do this, the relationship between the complete elliptic integral of the first kind and the arithmetic-geometric mean as well as Legendre's formula to approximately obtain this mean are used.
\end{abstract}

Keywords: Nonlinear oscillator; Approximate solutions; Duffing-harmonic oscillator; Chebyshev polynomials; Elliptic integrals; Arithmetic-geometric mean 


\section{Introduction}

Considerable attention has been directed towards the study of strongly nonlinear oscillators and several methods have been used to find approximate solutions to nonlinear oscillators [110]. In general, given the nature of a nonlinear phenomenon, the approximate methods can only be applied within certain ranges of the physical parameters and to certain classes of problems.

The purpose of this paper is to calculate analytical approximations to the periodic solutions to the Duffing-harmonic oscillator. This oscillator is a conservative non-linear oscillatory system modelled by a potential having a rational form for the potential energy [11]. To do this, the Chebychev series expansion of the restoring force is used [12-14] and the original nonlinear differential equation is approximated by a Duffing equation in which the coefficients for the linear and cubic terms depend on the initial amplitude, $A$. The replacement of the original nonlinear equation by an approximate Duffing equation allows us to obtain an approximate frequency-amplitude relation as a function of the complete elliptic integral of the first kind. As we can see, the results presented in this paper reveal that the method considered here is very effective and convenient for the Duffing-harmonic oscillator. Finally, we present a closed-form expression for the approximate frequency in terms of elementary functions. This expression is based on the relationship between the complete elliptic integral of the first kind and the arithmetic-geometric mean and the last one is approximately obtained using Legendre's formula.

\section{Solution procedure}

The governing non-dimensional equation of motion for the Duffing-harmonic oscillator is given as follows [11]

$$
\frac{\mathrm{d}^{2} x}{\mathrm{~d} t^{2}}+\frac{x^{3}}{1+x^{2}}=0
$$

with the initial conditions

$$
x(0) \doteq A \quad \text { and } \quad \frac{\mathrm{d} x}{\mathrm{~d} t}(0)=0
$$


Eq. (1) is a conservative nonlinear oscillatory system having a rational form for the non-dimensional restoring force. It has been demonstrated that all the curves in the phasespace corresponding Eq. (1) are closed, an all motions for arbitrary initial conditions give periodic solutions [11]. We denote the angular frequency of these oscillations by $\omega$ and one of our major tasks is to determine $\omega$ as a function of the initial amplitude $A$. For small values of the displacement $x$, the restoring force $F$ does have a dominant term proportional to $x$ and Eq. (1) approximates that of a truly Duffing-type nonlinear oscillator $\frac{\mathrm{d}^{2} x}{\mathrm{~d} t^{2}}+x^{3}=0$, whose angular frequency is $\omega \approx 0.84721 A$ [11], which tends to zero when $A$ decreases. While for large values of $x$, Eq. (1) approximates that of a linear harmonic oscillator $\frac{\mathrm{d}^{2} x}{\mathrm{~d} t^{2}}+x=0$, so, for large $A$, we have $\omega \approx 1$. Consequently the angular frequency $\omega$ increases from 0 to 1 as the initial value of $x(0)=A$ increases.

Eq. (1) is not amenable to exact treatment and, therefore, approximate techniques must be resorted to. In order to approximately solve this equation by means a cubication procedure based on the papers of Denman [12] and Jonckheere [13], in which the nonlinear restoring force is expanded in terms of the Chebyshev polynomials instead of in a Taylor series. To do we first introduce a reduced variable $[y=x / A$ in Eqs. (1) and (2)

$$
\begin{gathered}
\frac{\mathrm{d}^{2} y}{\mathrm{~d} t^{2}}+\frac{1}{A} f(y)=0, \\
y(0)=1 \text { and } \frac{A^{3} y^{3}}{1+A^{2} y^{2}} \\
\frac{\mathrm{d} y}{\mathrm{~d} t}(0)=0 .
\end{gathered}
$$

It is well known that it is possible to expand a function $f$ in terms of powers of $x$ (Taylor series) but also using other type of expansions. Denman [12] and Jonckheere [13] proposed the determination of the period of nonlinear oscillators by means the Chebyshev polynomials. Taking this into account, it is possible to expand the function $f(y)$ in terms of Chebyshev polynomials of the first kind $\left[T_{n}(x)\right]$ as follows [13] 


$$
f(y)=\sum_{n=0}^{\infty} b_{2 n+1}(A) T_{2 n+1}(y)
$$

where the first polynomials are [13]

$$
T_{1}(y)=y, T_{3}(y)=4 y^{3}-3 y, T_{5}(y)=16 y^{5}-20 y^{3}+5 y, \ldots
$$

and [13]

$$
b_{2 n+1}(A)=\frac{2}{\pi} \int_{-1}^{+1}\left(1-y^{2}\right)^{-1 / 2} f(y) T_{2 n+1}(y) \mathrm{d} y
$$

which are amplitude dependent. In Eq. (5) we have taken into account that $f(x)$ is an odd function of $x$. Substituting Eq. (5) into Eq. (3) it follows that

$$
\frac{\mathrm{d}^{2} y}{\mathrm{~d} t^{2}}+\frac{1}{A} \sum_{n=0}^{\infty} b_{2 n+1}(A) T_{2 n+1}(y)=0
$$

We can approximate Eq. (5) retaining only a finite number of terms and then different approximate equations to Eq. (3) can be obtained

$$
\frac{\mathrm{d}^{2} y}{\mathrm{~d} t^{2}}+\frac{1}{A} \sum_{n=0}^{N} b_{2 n+1}(A) T_{2 n+1}(y) \approx 0
$$

If only the first term $(N=0)$ is retained in Eq. (9), the nonlinear differential equation can be then approximated by the linear differential equation $\frac{\mathrm{d}^{2} y}{\mathrm{~d} t^{2}}+\frac{b_{1}(\dot{A}) T_{1}(y)}{A} \approx 0$. ('linearization' method [14]). A series expansion in terms of the Chebyshev polynomials converges much faster than the Taylor expansion [13] and due to this we can obtain a better approximation to Eq. (5) if we retaining the first two terms $(N=1$ in Eq. (9)) as follows 


$$
\frac{\mathrm{d}^{2} y}{\mathrm{~d} t^{2}}+\frac{b_{1}(A)}{A} T_{1}(y)+\frac{b_{3}(A)}{A} T_{3}(y) \approx 0 .
$$

where

$$
\begin{aligned}
f(y) & =\frac{A^{3} y^{3}}{1+A^{2} y^{2}} \approx b_{1}(A) T_{1}(y)+b_{3}(A) T_{3}(y)=\left[b_{1}(A)-3 b_{3}(A)\right] y+4 b_{3}(A) y^{3} \\
& =\frac{b_{1}(A)-3 b_{3}(A)}{A} x+\frac{4 b_{3}(A)}{A^{3}} x^{3}=\alpha(A) x+\beta(A) x^{3}
\end{aligned}
$$

and

$$
\begin{aligned}
& b_{1}(A)=\frac{2}{\pi} \int_{-1}^{+1}\left(1-y^{2}\right)^{-1 / 2} \frac{A^{3} y^{3}}{1+A^{2} y^{2}} T_{1}(y) \mathrm{d} y=A-\frac{2}{A}+\frac{2}{A \sqrt{1+A^{2}}} \\
& b_{3}(A)=\frac{2}{\pi} \int_{-1}^{+1}\left(1-y^{2}\right)^{-1 / 2} \frac{A^{3} y^{3}}{1+A^{2} y^{2}} T_{3}(y) \mathrm{d} y=\frac{2}{A}+\frac{8}{A^{3}}-\frac{2}{A^{3} \sqrt{1+A^{2}}}-\frac{6 \sqrt{1+A^{2}}}{A^{3}} \\
& \alpha(A)=\frac{b_{1}(A)-3 b_{3}(A)}{A}=1+\frac{4}{A^{2}}\left(\frac{5}{\sqrt{1+A^{2}}}-2\right)+\frac{24}{A^{4}}\left(\frac{1}{\sqrt{1+A^{2}}}-1\right) \\
& \beta(A)=\frac{4 b_{3}(A)}{A^{3}}=\frac{8}{A^{4}}\left(1-\frac{3}{\sqrt{1+A^{2}}}\right)+\frac{32}{A^{6}}\left(1-\frac{1}{\sqrt{1+A^{2}}}\right)
\end{aligned}
$$

The nonlinear differential equation in (1) is then approximated by the Duffing differential equation

$$
\frac{\mathrm{d}^{2} x}{\mathrm{~d} t^{2}}+\alpha(A) x+\beta(A) x^{3}=0
$$

in which $\alpha(A)]$ and $[\beta(A)$ depend on the initial amplitude $A$. In Figure 1 we have plotted $\alpha(A)$ and $\beta(A)$ as a function of $A$ por $0 \leq A \leq 5$. From Eqs. (14) and (15) it is easy to verify that

$$
\lim _{A \rightarrow 0} \alpha(A)=0, \quad, \lim _{A \rightarrow 0} \beta \dot{(A)=1}
$$




$$
\lim _{A \rightarrow \infty} \alpha(A)=1, \quad \quad \lim _{A \rightarrow \infty} \beta(A)=0 .
$$

and $\alpha(A) \geq 0$ and $\beta(A) \geq 0$ for all values of $A$.

As the cubication procedure consists in approximating the nonlinear differential (1) by Eq. (16) — which is the nonlinear differential equation for the Duffing oscillator-, the approximate frequency and solution for the initial equation will be the exact frequency and the exact solution for the Duffing equation $[3,4]$, which are given as follows

$$
\omega_{a}(A)=\frac{\pi \sqrt{\alpha+\beta A^{2}}}{2 K(m)}=\frac{\pi \sqrt{8+8 A^{2}+A^{4}+A^{6}-\left(8+4 A^{2}\right) \sqrt{1+A^{2}}}}{2 A^{2} \sqrt{1+A^{2}} K\left(\frac{4\left(4+3 A^{2}\right)-4\left(4+A^{2}\right) \sqrt{1+A^{2}}}{8+4 A^{2}-\left(8+A^{4}\right) \sqrt{1+A^{2}}}\right)} \text {. }
$$

$$
m=\frac{\beta A^{2}}{2\left(\alpha+\beta A^{2}\right)}=\frac{4\left(4+3 A^{2}\right)-4\left(4+A^{2}\right) \sqrt{1+A^{2}}}{8+4 A^{2}-\left(8+A^{4}\right) \sqrt{1+A^{2}}}
$$

$$
x_{a}(t)=A \operatorname{cn}\left[t \sqrt{\alpha+\beta A^{2}} ; m\right]
$$

where $\alpha$ and $\beta$ are given in Eqs. (14) and (15), $\operatorname{cn}\left[t \sqrt{\alpha+\beta A^{2}} ; m\right]$ is the Jacobi elliptic function and $K(m)$ is the complete elliptic integral of the first kind defined as follows

$$
K(m)=\int_{0}^{\pi / 2} \frac{\mathrm{d} \theta}{\sqrt{1-m \sin ^{2} \theta}}
$$

\section{Comparison with the exact and other approximate solution}

We illustrate the accuracy of the approach by comparing the approximate solutions previously obtained with the exact frequency $\left[\omega_{e^{\prime}}(A)\right]$ and the exact solution $\left[x_{\left.e^{(}(t)\right]}\right.$ of Eq. (1). Calculation of the exact angular frequency, $\omega_{e}(A)$; proceeds as follows. By integrating Eq. (1) and using the initial conditions in Eq. (2), we arrive at $[15,16]$ 


$$
\omega_{e}(A)=\frac{\pi}{2}\left[A \int_{0}^{1} \frac{\mathrm{d} u}{\left.\sqrt{A^{2}\left(1-u^{2}\right)+\log \left[\left(1+A^{2} u^{2}\right) /\left(1+A^{2}\right)\right.}\right]}\right]^{-1}
$$

For small values of the amplitude $A$ it is possible to take into account the following power series expansions $[15,16]$

$$
\begin{gathered}
\omega_{e}(A) \approx \frac{\pi}{2 K(1 / 2)} A-\ldots=0.847213 A-\ldots \\
\omega_{a}(A) \approx \frac{\Gamma^{2}(3 / 4)}{\sqrt{\pi}} A-\ldots=\frac{\pi}{2 K(1 / 2)} A-\ldots=0.847213 A-\ldots
\end{gathered}
$$

For very large values of the amplitude $A$ it is possible to take into account the following power series expansions $[15,16]$

$$
\begin{aligned}
& \omega_{e}(A) \approx 1-\frac{1}{A^{2}}+\ldots \\
& \omega_{a}(A) \approx 1-\frac{1}{A^{2}}+. .
\end{aligned}
$$

Furthermore, we have the following equations

$$
\lim _{A \rightarrow 0} \frac{\omega_{a}(A)}{\omega_{e_{e}}(A)}=1, \quad \lim _{A \rightarrow \infty} \frac{\omega_{a}(A)}{\omega_{e_{e}}(A)}=1
$$

It is important to point out that the exact behaviour of the approximate frequency when $A$ tends to zero is not obtained when other approximate methods as used including the harmonic balance method [17-19], the homotopy perturbation method [15,16], the energy balance method [20], the variational iteration method [21], a modified iteration procedure [22] or the Ritz procedure [23].

In Figure 2 we plotted the relative error for the approximate frequency $\left[\omega_{a}(A)\right]($ Eq. (19)). For comparison, we have also plotted the relative errors for the approximate frequencies $\left[\omega_{H P M_{2}}(A)\right]$ and $\left[\omega_{L W_{2}}(A)\right.$ obtained by Beléndez et al [16] and by Lim and Wu 
[17] using the second-order homotopy perturbation method (HPM) and the second-order harmonic balance method (HBM), respectively. As we can see from Figure 2, the relative errors for $-\omega_{a} \dot{i}(A)$ are lower than $0.071 \%$ (which is the maximum value for the relative error and corresponds to the oscillation amplitude $A=1.1$ ) for all the range of values of amplitude of oscillation $A$, and these relative errors tend to zero not only when $A$ to infinity as those for other approximate frequencies, but also when $A$ tends to zero (see Eq. (28)). Figure 2 indicates that $\left[\omega_{a}(A)\right]$ provides excellent approximations to the exact frequency $\left[\omega_{e}(A)\right]$ for the whole range of values of oscillation amplitude and reproduce the behaviour of the exact frequency not only for large values of the oscillation amplitude $A$ but also for small values of $A$.

The exact periodic solutions $x_{e}(t)$ achieved by numerically integrating Eq. (1), and the proposed normalized approximate periodic solution $x_{a}(t)$ in Eq. (21) for one complete cycle are plotted in Figures 3, 4, 5 and 6 for oscillation amplitudes $A=0.5,1.1$ (value of $A$ for which the relative error for the approximate frequency $\omega_{a}$ is maximum), 5 and 50 . In this figure parameter $h$ is defined as follows $: h=2 \pi \dot{t} / \omega_{e}(A)$. All these figures show that Eq. (21) provides a good approximation to the exact periodic solution. The results presented here illustrated very good agreement of the approximate frequency, $\omega_{a}(A)$, obtained in this paper using a cubication procedure, with the exact frequency $\omega_{e}(A):$ It is clear that the result obtained in this paper is better than those obtained previously by other authors.

\section{A closed-form expression for the approximate frequency in terms of elementary functions}

The approximate frequency in Eq. (19) is expressed in term of the complete elliptic integral of the first kind, $K(m)$. However, it is also possible to express the approximate frequency in a closed-form in terms of elementary functions. To do this we take into account that the complete elliptic integral of the first kind $K(m)$ (Eq. (22)) cannot be expressed in terms of elementary functions, but can be numerically evaluated with high precision by a simple procedure based on the arithmetic-geometric mean because the arithmetic-geometric mean is the basis of Gauss' method for the calculation of elliptic integrals [24-26]. Because the 
convergence of the arithmetic-geometric means is quadratic, an agreement of about $2^{n}$ digits between the means is expected after $n$ iterations [24].

The arithmetic-geometric mean of two numbers $p$ and $q, M(p, q)$ can be expressed in closed form in terms of the complete elliptic integral of the first kind as [25]

$$
M(p, q)=\frac{(p+q) \pi}{4 K\left(\frac{p-q}{p+q}\right)}
$$

which allows us to write $K(m)$ in Eq. (22) as follows [24,25]

$$
K(m)=\frac{\pi}{2 M(1, \sqrt{1-m})}
$$

The Legendre form of the arithmetic-geometric mean is given by [25]

$$
M(1, x)=\prod_{n=0}^{\infty} \frac{1}{2}\left(1+k_{n}\right)
$$

where $k_{0} \equiv x$ and $k_{n+1}=2 \sqrt{k_{n}} /\left(1+k_{n}\right)$. From Eq. (31) it is possible to approximately calculated the arithmetic-geometric mean by choosing a finite number of terms in this equation as follows

$$
M(1, x) \approx \prod_{n=0}^{\dot{N}} \frac{1}{2}\left(1+k_{n}\right)
$$

From Eqs. (19), (30) and (32) we obtain the following expression for the approximate frequency of the Duffing-harmonic oscillator 
$\omega_{a}^{a g m}(A)=\frac{1}{4 A^{2}}\left[\left(-8-4 A^{2}+A^{4}+8 \sqrt{1+A^{2}}\right)^{1 / 4}+\left(\frac{8+8 A^{2}+A^{4}+A^{6}-4\left(1+A^{2}\right) \sqrt{1+A^{2}}}{1+A^{2}}\right)^{1 / 4}\right]^{2}$

where only two iterations $(N=1)$ have been considered in Eq. (32)

$M(1, x) \approx \prod_{n=0}^{2} \frac{1}{2}\left(1+k_{n}\right)=\frac{1}{8}\left(1+k_{0}\right)\left(1+k_{1}\right)=\frac{1}{8}\left(1+k_{0}\right)\left(1+\frac{2 \sqrt{k_{0}}}{1+k_{0}}\right)=\frac{1}{8}(1+x+2 \sqrt{x})$

where $k_{0} \equiv x=\sqrt{1-m}$ and $m$ is given in Eq. (20).

For small values of the amplitude $A$ it is possible to take into account the following power series expansion

$$
\omega_{a}^{a g m}(A) \approx \frac{1}{16}\left(2+2^{3 / 4}\right)^{2} A-\ldots=0.847225 A-\ldots
$$

and for very large values of the amplitude $A$ it is possible to take into account the following power series expansion

$$
\omega_{a}^{a g m}(A) \approx 1-\frac{1}{A^{2}}+\ldots
$$

Furthermore, we have the following equations

$$
\lim _{A \rightarrow 0} \frac{\omega_{a}^{a g m}(A)}{\omega_{e}(A)}=1.000014, \quad \lim _{A \rightarrow \infty} \frac{\omega_{a}^{a g m}(A)}{\omega_{e}(A)}=1
$$

The maximum relative error for the frequency given in Eq. (33) is also $0.071 \%$ as the frequency given in Eq. (19) and it is also obtained for $A=1.1$. 


\section{Conclusions}

A cubication method for the Duffing-harmonic oscillator on the Chebyshev series expansion of the restoring force considered previously by Jonckheere [13] has been analyzed and discussed and an approximate frequency-amplitude relationship has been obtained. In this procedure, instead of approximately solve the original nonlinear differential equation, this one is replaced with a Duffing equation which is exactly solved. Excellent agreement between the approximate frequency and the exact one has been demonstrated. The discrepancy of this approximate frequency with respect to the exact one is as low as $0.071 \%$ and tends to zero not only when $A$ tends to infinity but also when $A$ tends to zero. This last behaviour can't be reproduced using other approximate methods commonly used. We think that this cubication method has great potential and can be applied to other strongly nonlinear oscillators with non-polynomial terms. Finally, using the relationship between the complete elliptical integral of the first kind and the arithmetic-geometric mean a closed-form expression for the approximate frequency is obtained in terms of elementary functions. As Carvalhaes and Supes pointed out, 'this approach is not new, but is not widely known' [24].

\section{Acknowledgements}

This work has been supported by the "Ministerio de Ciencia e Innovación" of Spain, under projects FIS2008-05856-C02-01 and FIS2008-05856-C02-02. 


\section{References}

[1] A. H. Nayfeh, Problems in Perturbations (Wiley, New York, 1985)

[2] J. H. He, "Modified Lindstedt-Poincare methods for some non-linear oscillations. Part III: double series expansion”, Int. J. Nonlinear Sci. Numer. Simul. 2, 317-320 (2001).

[3] R. E. Mickens, Oscillations in Planar Dynamics Systems (World Scientific, Singapore, 1996)

[4] P. Amore, A. Raya and F. M. Fernández, "Alternative perturbation approaches in classical mechanics", Eur. J. Phys. 26, 1057-1063 (2005).

[5] P. Amore and A. Aranda, "Improved Lindstedt-Poincaré method for the solution of nonlinear problems", J. Sound. Vib. 283, 1115-1136 (2005).

[6] M. T. Darvishi, A. Karami and B. C. Shin, “Application of He's parameter-expansion method for oscillators with smooth and odd nonlinearities", Phys. Lett. A 372, 53815384 (2008).

[7] J. H. He, "Variational approach for nonlinear oscillators", Chaos, Solitons \& Fractals 34, 1430-1439 (2007).

[8] T. Özis and A. Yildirim, “A comparative study of He's homotopy perturbation method for determining frequency-amplitude relation of a nonlinear oscillator with discontinuities", Int. J. Nonlinear Sci. Numer. Simul. 8, 243-248 (2007).

[9] A. Beléndez, A. Hernández, T. Beléndez, M. L. Álvarez, S. Gallego, M. Ortuño and C. Neipp, “Application of the harmonic balance method to a nonlinear oscillator typified by a mass attached to a stretched wire", J. Sound Vib. 302, 1018-1029 (2007).

[10] J. H. He, "Some asymptotic methods for strongly nonlinear equations", Int. J. Mod. Phys. B 20, 1141-1199 (2006).

[11] R. E. Mickens, "Mathematical and numerical study of the Duffing-harmonic oscillator”, J. Sound Vib. 244, 563-567 (2000). 
[12] J. H. Denman, "An approximate equivalent linearization technique for nonlinear oscillations", J. Appl. Mech. 36, 358-360 (1969).

[13] R. E. Jonckheere, "Determination of the period of nonlinear oscillations by means of Chebyshev polynomials", ZAMM-Zeitschrift fur Angewandte Mathematik und Mechanik 55, 389-393 (1971).

[14] A. Beléndez, M. L. Álvarez, E. Fernández and I. Pascual, "Linearization of conservative nonlinear oscillators", Eur. J. Phys. 30, 259-270 (2009).

[15] A. Beléndez, A. Hernández, T. Beléndez, E. Fernández, M. L. Álvarez y C. Neipp, "Application of He's homotopy perturbation method to the Duffing-harmonic oscillator”, Int. J. Nonlinear Sci. Numer. Simul. 8, 79-88 (2007).

[16] A. Beléndez, C. Pascual, E. Fernández, C. Neipp and T. Beléndez, "Higher-order approximate solutions to the relativistic and Duffing-harmonic oscillators by modified He's homotopy methods", Phys. Scr. 77, art. 025004 (14 pp) (2008). DOI: 10.1088/0031-8949/77/02/025004

[17] C. W. Lim and B. S. Bu, "A new approach to the Duffing-harmonic oscillator", Phys. Lett. A $311365-373$ (2003).

[18] H. Hu and J. H. Tang, "Solution of a Duffing-harmonic oscillator by the method of harmonic balance", J. Sound Vib. 294, 637-639 (2006).

[19] C. W. Lim, B. S. Wu and W. P. Sun, "Higher accuracy analytical approximations to the Duffing-harmonic oscillator”, J. Sound Vib. 296, 1039-1045 (2006).

[20] T. Özis and A. Yildirim, "Determination of the frequency-amplitude relation for a Duffing-harmonic oscillator by the energy balance method", Computers and Mathematics with Applications 54, 1184-1187 (2007).

[21] M. Fesanghary, T. Pirbodaghi, M. Asghari and H. Sojoudi, "A new analytical approximation to the Duffing-harmonic oscillator", Chaos, Solitons \& Fractals (2009), doi:10.1016/j.chaos.2009.01.024. 
[22] H. Hu, "Solution of the Duffing-harmonic oscillator by an interation procedure", $J$. Sound Vib. 298, 446-452 (2006).

[23] S. B. Tiwari, B. Nageswara Rao, N. Shivakumar Swamy, K. S. Sai and H. R. Nataraja, “Analytical study on a Duffing-harmonic oscillator”, J. Sound Vib. 285, 1217-1222 (2005).

[24] C. G. Carvalhaes and P. Suppes, "Approximation for the period of the simple pendulum based on the arithmetic-geometric mean", Am. J. Phys 76, 1150-1154 (2008).

[25] E. W. Weisstein, “Arithmetic-Geometric Mean”. From MathWorld--A Wolfram Web Resource. http://mathworld.wolfram.com/Arithmetic-GeometricMean.html

[26] E. Salamin, "Computation of $\pi$ using arithmetic-geometric mean", Mathematics in Computation 30, 565-570 (1976). 


\section{Figure captions}

Figure 1.- Coefficients for the linear ( $\bar{\alpha})$ and cubic $(\bar{\beta}])$ terms of the approximate Duffing equation as a function of the oscillation amplitude.

Figure 2.- Relative errors for approximate frequency obtained in this paper (CUB) and the second-order approximate frequencies obtained using the homotopy perturbation method (HPM) and the harmonic balance method (HBM).

Figure 3.- Comparison of the approximate solution, Eq. (21) (dashed line and triangles) with the numerical exact solution (continuous line and circles) for $A=0.5$.

Figure 4.- Comparison of the approximate solution, Eq. (21) (dashed line and triangles) with the numerical exact solution (continuous line and circles) for $A=1.1$.

Figure 5.- Comparison of the approximate solution, Eq. (21) (dashed line and triangles) with the numerical exact solution (continuous line and circles) for $A=5$.

Figure 6.- Comparison of the approximate solution, Eq. (21) (dashed line and circles) with the numerical exact solution (continuous line and circles) for $A=50$. 